\title{
Coupled-rearrangement-channels calculation of the three-body system under the absorbing boundary condition
}

\author{
M. Iwasaki ${ }^{1}$, R. Otani ${ }^{1}$, M. Ito ${ }^{1, a}$, and M. Kamimura ${ }^{2}$ \\ ${ }^{1}$ Department of Pure and Applied Physics, Kansai University, 3-3-35 Yamatecho Suita, Osaka, Japan \\ ${ }^{2}$ RIKEN Nishina Center for Accelerator-based Science, RIKEN, Wako, Saitama, Japan
}

\begin{abstract}
We formulate the absorbing boundary condition (ABC) in the coupledrearrangement-channels variational method (CRCVM) for the three-body problem. The absorbing potential is introduced in the system of the identical three-bosons, on which the boson symmetry is explicitly imposed by considering the rearrangement channels. The resonance parameters and the strength of the monopole breakup are calculated by the $\mathrm{CRCVM}+\mathrm{ABC}$ method, and the results are compared with the complex scaling method (CSM). We have found that the results of the ABC method are consistent with the CSM results. The effect of the boson symmetry, which is often neglected in the calculation of the triple $\alpha$ reactions, is also discussed.
\end{abstract}

\section{Introduction}

One of the current issues in nuclear physics is the structure of the borromean three-body systems, which mainly appear around Neutron- or Proton-drip lines and its dynamics of continuum states above the particle decay thresholds [1]. The borromean systems around the drip line are weakly binding systems. Since the particle thresholds of the borromean nuclei open at the lower excitation energy than the normal nuclei, a large part of the excited states is embedded in continuum region, which is observed through the breakup reaction, for instance. Therefore, it is very important to describe the structural changes of the borromean systems as well as its breakup dynamics in a consistent manner.

In a naive treatment of the unbound states, a scattering boundary condition should be imposed among the interacting particles, but imposing the scattering boundary condition for three particles is complicated in general. In particular, the exact treatment of the three-body (or few-body) scattering state requires special techniques for the long range interaction, such as the Coulomb force [2]. In order to avoid the complexity of the three-body scattering boundary condition, the scattering problem is often reduced to the bound-state-like problem by transforming the Hamiltonian of a total system into a non-Hermite form. There are two representative methods of the non-Hermitian transformations: the complex scaling method (CSM) [3-5] and the method of the absorbing boundary condition (ABC), or the method of the complex absorbing potential (CAP) [6-11].

In CSM, the radial coordinates and the respective momenta in the Hamiltonian of the total system is simply rotated with the angle of $\theta$ in the complex plane, such as $r \rightarrow r e^{i \theta}$ and $k \rightarrow k e^{i \theta}$, while, in

\footnotetext{
ae-mail: itomk@kansai-u.ac.jp
} 
$\mathrm{ABC}$, the negative imaginary potential is placed at the outer region of the system. These two procedures commonly give rise to the damping of the wave function at the asymptotic region. Therefore, the three-body scattering problem can be transformed into the bound-state-like problem, in which the variational calculation using the basis expansion technique is available.

There are many sophisticated applications of CSM to the reaction problem of the three-body continuum states [3, 4]. For example, the CSM calculations are greatly successful in describing the Coulomb breakup into the three-body continuum, such as ${ }^{6} \mathrm{He} \rightarrow \alpha+N+N$ [3]. In the CSM calculations, the rearrangement of the Jacobi coordinates among the three particles, is completely taken into account. The variational calculation with the rearrangement channels is called the coupledrearrangement-channels variational method (CRCVM), and the CRCVM treatment is essential to obtain the rapid convergence of the binding energy in the three-body system [12].

The application of the ABC method to the three-body problem is still limited although the CSM + CRCVM calculation has already been established in the three-body problem. For example, there are the calculations of the ABC method in the schematic three-body system [7] and the nuclear breakup induced by the deuteron scattering [8], but no coordinate rearrangement is considered in these calculations. This shortcoming in the ABC calculation is because the formulation of the ABC method in CRCVM still remains unclear in contrast to the CSM treatment, in which all the coordinates and momenta are uniformly rotated by the common scaling angle.

In the $\mathrm{ABC}$ method, the negative imaginary potentials should included, which are the function of the relative coordinate of the decaying particles. In the single coordinate systems, such as twobody systems, the absorbing potential is uniquely determined, but, in the three-body system with the rearrangement channels, the way to introduce the absorber is not so definite. In order to establish the $\mathrm{ABC}$ formalism in CRCVM, we should carefully test the possible ways of the absorbing potential.

Since the application of CSM is difficult in some special problems [10], it is important to develop the $\mathrm{ABC}$ method in the CRCVM treatment. In the present report, we present the formulation of the CRCVM + ABC method in the identical three-boson system and demonstrate that the present formulation nicely works in the three-body problem with the rearrangement channels. Here we focus on the calculation of the resonance parameters and the breakup strength induced by the monopole external field. The results of the ABC method are compared with the CSM calculation, and the validity of the ABC method is discussed. Moreover, we investigate the effect of the boson symmetry on the strength function because the boson symmetry has often been neglected in the calculation of the reaction rate of the triple $\alpha$ reaction $[13,14]$. In the precise evaluation of the reaction rate, the symmetrization effect may be crucial, and we check the validity of the neglect of the boson symmetry.

\section{Framework}

\subsection{Three-body system with the absorbing boundary condition}

In the present calculation, we handle the three-boson system, which has the following total Hamiltonian,

$$
\hat{H}=-\frac{\hbar^{2}}{2 \mu_{\mathbf{r}}} \nabla_{\mathbf{r}}^{2}-\frac{\hbar^{2}}{2 \mu_{\mathbf{R}}} \nabla_{\mathbf{R}}^{2}+\sum_{i>j} V\left(s_{i j}\right),
$$

where, the first two terms represent the kinetic energies with the Jacobi coordinate of $\mathbf{r}$ and $\mathbf{R}$, which denote the relative coordinate of the first and second particles (1-2) and the coordinate between the center of mass of $1+2$ particles and the third particle $((1+2)-3)$, respectively. $\mu_{\mathbf{r}}$ and $\mu_{\mathbf{R}}$ represent the reduced masses respective to the Jacobi coordinate. In the present calculations, a kinematic factor is set to be $\hbar^{2} / m=1 / 2\left(\mathrm{MeV} \cdot \mathrm{fm}^{2}\right)$ for simplicity. 
The last term denotes the two-body potential of the $i-j$ particles with relative distance $s_{i j}$, which is assumed to be the Gaussian potential [7], such as

$$
V(s)=-2 e^{-0.16 s^{2}}+e^{-0.04 s^{2}}
$$

In the $\mathrm{ABC}$ method, the total Hamiltonian is modified to include the negative imaginary potential $-i \hat{W}$ with a strength $\eta$, such as

$$
\hat{H} \rightarrow \hat{H}^{\eta}=\hat{H}-i \eta \hat{W} .
$$

Here we should set the functional form of the absorbing potential $\hat{W}$. In a naive treatment, the absorber should be included in accordance to the Jacobi coordinate in each of the rearrangement channel, such as

$$
\hat{W}=\sum_{c=1,2,3}\left(f\left(r_{c}-r_{a}\right)+f\left(R_{c}-R_{a}\right)\right) .
$$

with the form factor of the shifted polynomial function $[6,10]$,

$$
f\left(r_{c}-r_{a}\right)=\left(r_{c}-r_{a}\right)^{p} \theta\left(r_{c}-r_{a}\right) .
$$

$r_{c}$ and $R_{c}$ represent the absolute values of the Jacobi vectors in the rearrangement channel of $c$, while $r_{a}$ show the starting point of the absorber. In Eq. (4), the absorbing potentials are introduced along the Jacobi coordinate in the $c$-th rearrangement channels, and they are symmetrized on the channel suffix $c$. Another possible way in introducing the absorber is the imaginary potentials among the pair of two particles,

$$
\hat{W}=\sum_{i>j} f\left(s_{i j}-r_{a}\right),
$$

where $s_{i j}$ denotes the magnitude of the relative coordinate of the $i$-th and $j$-th particles. In Eqs. (4) and (6), we set $p=4$ and $r_{a}=R_{a}=14 \mathrm{fm}$, which are found to be an appropriate setting in the previous study of the two-body system [9-11].

We solve the Schrödinger equation for $\hat{H}^{\eta}$ in Eq. (3)

$$
\hat{H}^{\eta} \Psi_{n}^{\eta}(r, R)=E_{n}(\eta) \Psi_{n}^{\eta}(r, R) .
$$

The wave function $\Psi_{n}^{\eta}(r, R)$ and energy eigenvalue $E_{n}(\eta)$ for the $n$-th eigenstate depend on the strength of the absorber $\eta$. In the present calculation, the S-wave relative motion is assumed in $\Psi_{n}^{\eta}(r, R)$ and hence, it depends on the magnitudes of the Jacobi coordinates, $r$ and $R$. Eq. (7) is solved by the basis expansion method, in which the oscillating Gaussian basis is employed [5, 12]. In the wave function of $\Psi_{n}^{\eta}(r, R)$, the boson symmetry is exactly considered by introducing the rearrangement channels.

\subsection{Strength function}

We also calculate the strength function of the three-body breakup into the continuum. A general definition of the strength function is given by

$$
\mathcal{S}(E)=\sum_{v}\left|\left\langle\Psi_{v}\left|\hat{O}_{\lambda}\right| \Psi_{i}\right\rangle\right|^{2} \delta\left(E-E_{v}\right),
$$

where $\Psi_{i}$ and $\Psi_{v}$ are the initial bound state and the $v$-th final state having the energy eigenvalue $E_{v}$ in continuum, respectively. In Eq. (8), $\hat{O}_{\lambda}$ denotes the operator of an external field with the rank $\lambda$, and 
the monopole field $(\lambda=0)$ is considered, which are the squared particle coordinate measured from the center of mass for the total system, such as

$$
\hat{O}_{0}=\sum_{i=1,2,3} \xi_{i}^{2}
$$

where the vector $\xi_{i}$ represents the coordinate of the $i$-th particle measured from the center of mass for the total system.

The summation of the final state $\Psi_{v}$ can be eliminated from Eq. (8) by assuming that the final states of $\Psi_{v}$ forms a complete set of the Hamiltonian $H$. If the Hamiltonian is transformed into $H^{\eta}$ by switching on the absorber, we can get the following expression in the coordinate integration;

$$
\begin{aligned}
& \mathcal{S}_{\lambda}^{\eta}(E)=-\frac{1}{\pi} \mathfrak{J}\left[\mathcal{R}_{\lambda}^{\eta}(E)\right] \\
& \mathcal{R}_{\lambda}^{\eta}(E)=\left\langle\Psi_{i}\left|\hat{O}_{\lambda}^{\dagger} \mathcal{G}^{\eta}(E) \hat{O}_{\lambda}\right| \Psi_{i}\right\rangle=\iint D \mathbf{X} D \mathbf{X}^{\prime} \Psi_{i}^{*}\left(\mathbf{X}^{\prime}\right) \hat{O}_{\lambda}^{\dagger} \mathcal{G}^{\eta}\left(E, \mathbf{X}^{\prime}, \mathbf{X}\right) \hat{O}_{\lambda} \Psi_{i}(\mathbf{X}) .
\end{aligned}
$$

Here $\mathcal{R}_{\lambda}(E)$ is called the response function, which is represented by a set of the Jacobi coordinate of $\mathbf{X}=(\mathbf{r}, \mathbf{R})$ and $D \mathbf{X}=d \mathbf{r} d \mathbf{R}$. In Eq. (11), $\mathcal{G}^{\eta}$ denotes the Green function,

$$
\mathcal{G}^{\eta}\left(E, \mathbf{X}^{\prime}, \mathbf{X}\right)=\left\langle\mathbf{X}^{\prime}\left|\frac{1}{E-\hat{H}^{\eta}}\right| \mathbf{X}\right\rangle, \quad \mathcal{G}^{\eta}(E)=\frac{1}{E-\hat{H}^{\eta}},
$$

which is defined by the total Hamiltonian in Eq. (3), which includes the absorber with the strength $\eta$. The initial wave function of $\Psi_{i}$ is invariant with respect to the introduction of the absorber and hence, the superscript of $\eta$ is dropped in $\Psi_{i}$.

The calculation of the response function in Eq. (11) can be achieved by assuming the extended completeness relation (ECR) for the discrete solutions under the absorbing boundary,

$$
\hat{1}=\sum_{n}^{N}\left|\Psi_{n}^{\eta}><\tilde{\Psi}_{n}^{\eta}\right|=\sum_{n \in B}\left|\Psi_{n}^{\eta}><\tilde{\Psi}_{n}^{\eta}\right|+\sum_{n \in R}\left|\Psi_{n}^{\eta}><\tilde{\Psi}_{n}^{\eta}\right|+\sum_{n \in C}\left|\Psi_{n}^{\eta}><\tilde{\Psi}_{n}^{\eta}\right|
$$

Here $\Psi_{n}^{\eta}$ denotes the $n$-th solution of Eq. (7), which is obtained by the basis expansion method. In the last equation, the summation is decomposed into three parts: the bound states $(B)$, the resonant states $(R)$, and the non-resonant continuum states $(C)$. The final expression of the response function, on which the ECR is implemented, is given by

$$
\mathcal{R}_{\lambda}^{\eta}(E)=\sum_{n}^{N} \frac{<\Psi_{i}\left|\hat{O}_{\lambda}^{\dagger}\right| \Psi_{n}^{\eta}><\tilde{\Psi}_{n}^{\eta}\left|\hat{O}_{\lambda}\right| \Psi_{i}>}{E-E_{n}(\eta)}=\mathcal{R}_{\lambda B}^{\eta}(E)+\mathcal{R}_{\lambda R}^{\eta}(E)+\mathcal{R}_{\lambda C}^{\eta}(E)
$$

where the response function is decomposed into three parts in accordance with Eq. (13). This decomposition is very useful in the analysis of the reaction mechanism of the three-body breakup reaction $[4,11]$. For example, we can understand the dominant breakup process, such as the resonance decay or the direct breakup, in which the ground state directly goes into the continuum states. In this response function, the denominator contains a complex number of $E_{n}(\eta)$, which is the eigenvalue in the $\mathrm{ABC}$ solution to Eq. (7). Thus, this function is converged to a finite value even if the excitation energy $E$ is close to the real part of $E_{n}(\eta)$. 


\section{Results}

\subsection{Energy distribution and the resonance parameters}

Two panels in figure 1 show the distribution of the energy eigenvalues obtained by solving Eq. (7) for the identical three-bosons system under the absorbing boundary condition. The absorber is introduced in terms of the function of the Jacobi coordinate, which is shown in Eq. (4). The left panel is the result of the weak absorber $\left(\eta=2.0 \times 10^{-7}\right)$, while the right panel is that of the strong absorber $\left(\eta=2.0 \times 10^{-4}\right)$.
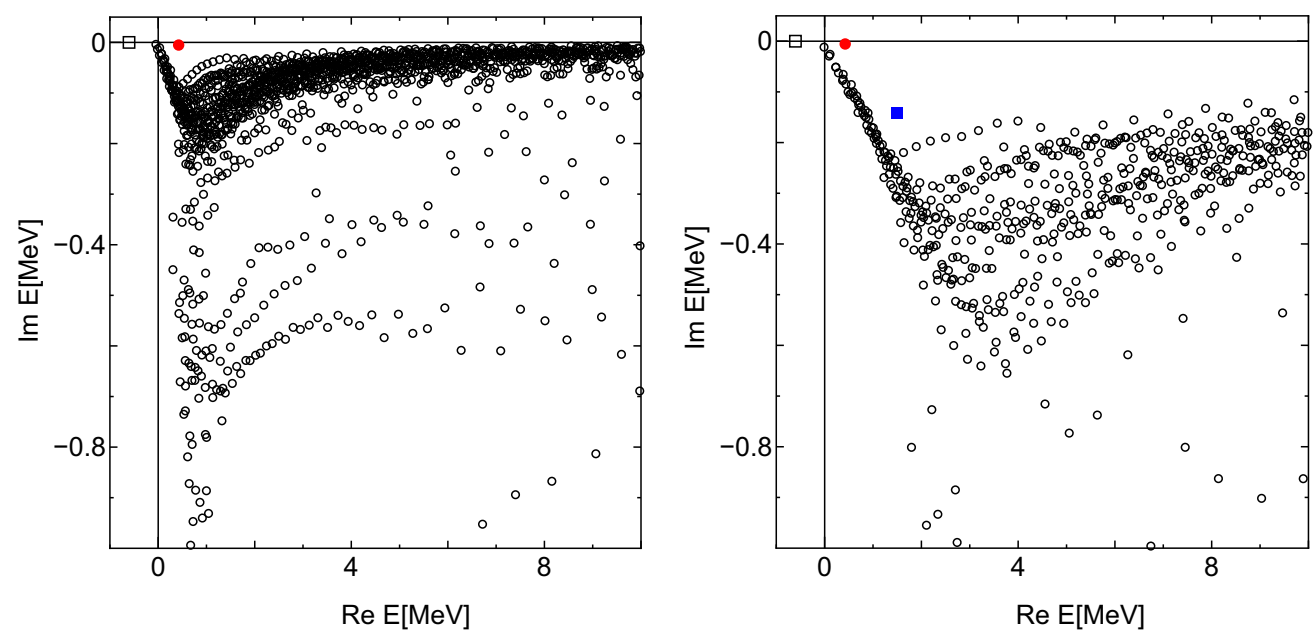

Figure 1. Distribution of the energy eigenvalues in the complex energy plane. The open square and the open circles show the bound state and the non-resonant continuum states, respectively. The solid circle and the solid square show the first and second resonances, respectively. The left panel shows the case of $\eta=2 \times 10^{-7}$ and the right panel is the case of $\eta=2 \times 10^{-4}$

If the absorbing potential is switched off, the eigenvalues are located along the real (horizontal) axis. The energy eigenvalues are distributed in the complex energy plane when the absorbing potential is activated. In the negative real energy region, there is a bound state (open square), which is invariant under the absorbing boundary. In the positive energy region, the resonant states are separated from the continuum spectra (open circles), and the separation depends on the strength of the absorber.

In the weak absorber case (left panel), the imaginary part of the continuum energy is not so large, and the continuum states are concentrated around the real axis. Therefore, in the weak absorber case, only one resonance (solid circle) is separated from the continuum states (open circles). On the contrary, the imaginary energy of the continuum states becomes larger when the strength of the absorber is strong, as shown in the right panel. This increased imaginary energy leads to the separation of the second resonance (solid square), as can be confirmed in the right panel.

When the absorber strength $\eta$ increases continuously, the imaginary energy of the continuum states monotonically increases, while the energies of the resonant states $E_{r e s}(\eta)$ are almost invariant after the resonances are separated from the continuum. Therefore, the stronger absorber is needed in order to obtain the broad resonant states with a large imaginary energy. 
We have also checked the energy distribution calculated from the other type of the absorbing boundary, which is imposed on the particle pair coordinate, shown in Eq. (6). We have confirmed the resultant energy distribution is almost the same as the distribution shown in Fig. 1.

Table 1. Resonance parameters for the first resonance calculated at the optimal strength $\eta_{o}$. The resonance energy $\left(E_{R}\right)$ and the resonance width $\left(\Gamma_{R}\right)$ are shown in unit of $\mathrm{MeV} .<W\left(\eta_{o}\right)>$ represent the expectation value of the absorber at $\eta_{o}$. In the second row, the result of the ABC method is shown, while the results of CSM is shown in the lowest row.

\begin{tabular}{|c|c|c|c|c|}
\hline Method & $E_{R}$ & $\Gamma_{R} / 2$ & $<W\left(\eta_{o}\right)>$ & $\eta_{o}$ \\
\hline ABC (Jacobi) & 0.424 & $5.239 \times 10^{-3}$ & $6.942 \times 10^{-7}$ & $1.78 \times 10^{-6}$ \\
\hline ABC (pair) & 0.424 & $5.239 \times 10^{-3}$ & $7.118 \times 10^{-7}$ & $8.0 \times 10^{-6}$ \\
\hline CSM & 0.424 & $5.260 \times 10^{-3}$ & $\mathrm{X}$ & $\mathrm{X}$ \\
\hline
\end{tabular}

Table 2. Same as the table. 1 except for the second resonance.

\begin{tabular}{|c|c|c|c|c|}
\hline Method & $E_{R}$ & $\Gamma_{R} / 2$ & $<W\left(\eta_{o}\right)>$ & $\eta_{o}$ \\
\hline ABC (Jacobi) & 1.500 & 0.141 & $4.475 \times 10^{-5}$ & $1.7 \times 10^{-4}$ \\
\hline ABC (pair) & 1.500 & 0.144 & $7.015 \times 10^{-5}$ & $6.3 \times 10^{-4}$ \\
\hline CSM & 1.498 & 0.142 & $\mathrm{X}$ & $\mathrm{X}$ \\
\hline
\end{tabular}

In Fig. 1, the strength of the absorber, $\eta$, is fixed to a constant value, but the energy eigenvalues of the resonance basically depend on the strength of $\eta$. Therefore, the strength parameter of $\eta$ should be fixed to be an optimal value $[6,9,10]$. Here we denote the resonance eigenvalue as a function of $\eta$ by $E_{r e s}(\eta)$. If the basis set is complete, the correct resonance parameters can be obtained as a limit of $\eta \rightarrow 0$. In the finite basis set calculations, however, a finite $\eta$ gives approximate resonance parameters. The condition for an optimal strength $\eta_{o}$ is

$$
\left|\eta \frac{d E_{r e s}(\eta)}{d \eta}\right|_{\eta=\eta_{o}}=\min
$$

Here the derivatives $\eta \cdot d E_{\text {res }}(\eta) / d \eta$ are obtained simply by employing the generalized HellmannFeynman theorem [15] like

$$
\left|\eta \frac{d E_{r e s}(\eta)}{d \eta}\right|=\eta\left|<\tilde{\Psi}_{r e s}^{\eta}(r, R)\right| \hat{W}\left|\Psi_{r e s}^{\eta}(r, R)>\right| \equiv<W(\eta)>,
$$

where $\Psi_{r e s}^{\eta}(r, R)$ represents the resonance wave function in Eq. (7) calculated with an absorber of the strength $\eta$. The tilde in the bra-state means that the complex conjugate is not taken in the radial integration.

An argument to justify the condition of Eq. (15) was presented on the basis of the asymptotic expansion of the function $E(\eta)$. [6, 7]. Let us discuss the optimization of $\eta$ in a simple argument. If $\eta$ is very small and the range of the basis function is limited to the shorter region, absorption occurs in region of large distance, where no basis functions exist. Then the resonant eigenvalue becomes almost real. As $\eta$ increases, the absorption can be efficiently described by a given basis set, and the 
imaginary part of $E_{r e s}(\eta)$ increases. When the spatial region of the dominant absorption sufficiently overlaps the region of the basis functions, the imaginary part of $E_{r e s}(\eta)$ will be close to the true width. If one further increases $\eta$, the reflection due to the absorbing potential becomes substantial, and this will cause the imaginary part of $E_{r e s}(\eta)$ to decrease. Therefore, there is an optimal strength, $\eta_{o}$, for a resonance, in which the reflection from the absorber and the incompleteness of the basis expansion balance each other. The stationary behavior of the resonance energy around $\eta=\eta_{o}$ have been deeply analyzed in Ref. [10].

The resonance parameters of $E_{R}$ and $\Gamma_{R}$ are calculated at the optimal strength of $\eta_{o}$ according to the relation of $E_{\text {res }}\left(\eta_{o}\right)=E_{R}-i \Gamma_{R} / 2$. The resonance parameters for the first resonance are summarized in table 1. In the second row from the top, the resonance parameters calculated from the Jacobi absorber are shown, while the parameters obtained from the pair absorber are shown in the third row. The results of the Jacobi absorber is same as those of the pair absorber. In both of the ABC results, the optimal strength $\eta_{o}$ is quite weak $\left(\sim 10^{-6}\right.$ in Jacobi and $\sim 10^{-5}$ in pair) and hence, the expectation value of $<W\left(\eta_{o}\right)>$ is also small $\left(\sim 10^{-7}\right)$ in comparison to the magnitude of the resonance parameters. These two results of $\mathrm{ABC}$ is consistent to the CSM calculation, shown in the bottom row.

We have done the similar optimization of $\eta$ for the second resonance, and the results are summarized in table 2. The resonance parameters of the Jacobi ABC method (second row) is almost same as the method of the pair coordinate (third row). The absorber strength for the second resonance $\left(\eta_{o} \sim\right.$ $\left.10^{-4}\right)$ is stronger than that for the first resonance $\left(\eta_{o} \sim 10^{-6}\right)$ because the strong absorber is needed to separate the broad resonance from the continuum. Therefore, the reflection effect from the absorber is much more prominent in the second resonance. Due to this reflection, the resonance parameters of $\mathrm{ABC}$ considerably deviates from the CSM calculation, shown at the bottom row.

\subsection{Strength function of the monopole breakup}

We discuss the application of the ABC method to the strength function of the three-body breakup. The strength function can be calculated accroding to the response function in Eq. (14), in which the ABC solutions in Fig. 1 are substituted into $\Psi_{n}^{\eta}$. Left panel in Fig. 2 shows the strength function from the ground state to the three-body continua, which is induced by the monopole external field. The solid curve shows the result of the ABC method with the Jacobi absorber, while the dashed curve shows the CSM result. The ABC calculation follows the curve obtained by the CSM calculation over all energy region although a small oscillation arise in the higher energy region of $E \geq 3 \mathrm{MeV}$, where the magnitude of the strength is smaller by about $10^{-6}$ than the peak magnitude at $E \sim 0.5 \mathrm{MeV}$. We have checked that the pair absorber gives the similar strength function to the result of the Jacobi absorber.

The sharp peak at $E \sim 0.5 \mathrm{MeV}$ is generated by the contribution from the first resonance, while the broad shoulder at $E \sim 1 \mathrm{MeV}$ is due to the existence of the second resonance. In order to see the intrinsic contributions in the strength function, the strength decomposition according to Eqs. (13) and (14) is shown in the right panel of Fig. 2. The contribution from the first resonance, which is plotted by the dashed curve, is predominant in the total strength, shown by the solid curve. The dotted curve represents the total contribution of the second resonance plus all of the non-resonant continua. The broad peak of the dotted curve correspond to the bump in the total strength, appearing just below $E \sim$ $1 \mathrm{MeV}$.

In the low energy region of $E \leq 0.3 \mathrm{MeV}$, the total strength is almost zero, but the individual contributions from the first resonance (dashed curve) and the other contribution (dotted curve) are finite value with the opposite signs. The total strength is constructed from the cancellation of these contributions. This feature of the cancellation is also seen in the strength function of the two-body breakup, which is studied in the CSM and ABC calculations $[3,4,11]$. 

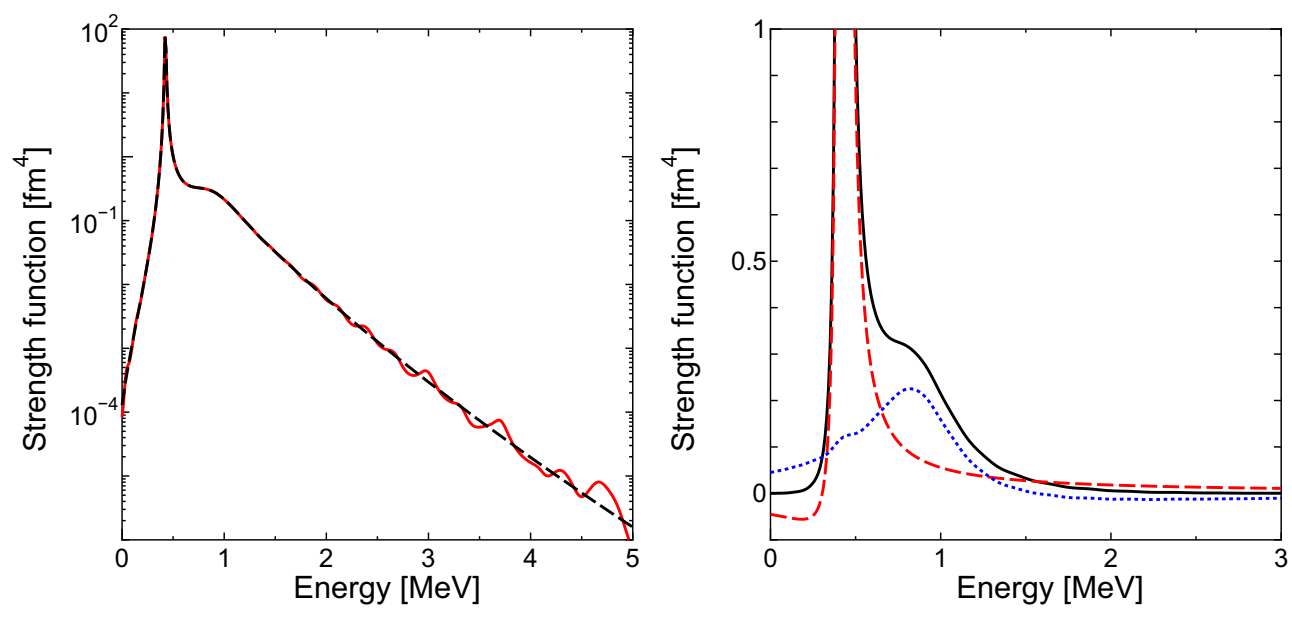

Figure 2. Left panel: Monopole strength functions calculated from the ABC and CSM results. The solid and dashed curves show the ABC and CSM results, respectively. Right panel: Decomposed strength function. The solid curve shows the total strength, while the dashed and dotted curves represent the contributions from the first resonance and the other resonance plus continuum. In these panels, the Jacobi absorber is used for the ABC calculation.

We have also done the decomposition of the strength function on the basis of the CSM treatment $[3,4]$, in which the application of ECR has already been established. The individual contributions, such as the resonance and the non-resonant continuum, in the strength function is common between $\mathrm{ABC}$ and CSM and hence, the ABC calculation is consistent to the CSM calculation.

\subsection{Effect of the boson symmetry}

Finally, we discuss the effect of the boson symmetry on the strength function, which is taken into account by the rearrangement channels. The effect of the rearrangement channels is investigated for the total binding energy in the three-body system [3, 12]. The inclusion of the rearrangement channel is found to be essential to obtain the rapid convergence of the binding energy, but there is no detailed analysis about the effect of the rearrangement channel on the strength of the three-body breakup reaction.

In the left panel of Fig. 3, the strength functions with and without the boson symmetry are compared. The former and latter results are plotted by the solid and dashed curves, respectively. These strengths are calculated by CSM. The overall shape of the strength is common in these two types of the calculations, but there is a clear difference in the peak structures and the magnitude.

In the result without the boson symmetry (dashed curve), a sharp spike appears just above $E \sim 0.5$ $\mathrm{MeV}$. This spike is generated from the resonant states with a sharp width, which should be excluded by the boson symmetry. Since the boson symmetry restricts the possible phase space among the three particles, the number of the resonances are enhanced in the case without the boson symmetry. Therefore, the neglect of the boson symmetry leads to the formation of the spurious peak structure in the strength function.

As for the magnitude of the strength, the boson symmetry gives a minor effect on the strength at the higher energy side of $E \geq 1 \mathrm{MeV}$, where a smooth energy dependence can be seen. However, 

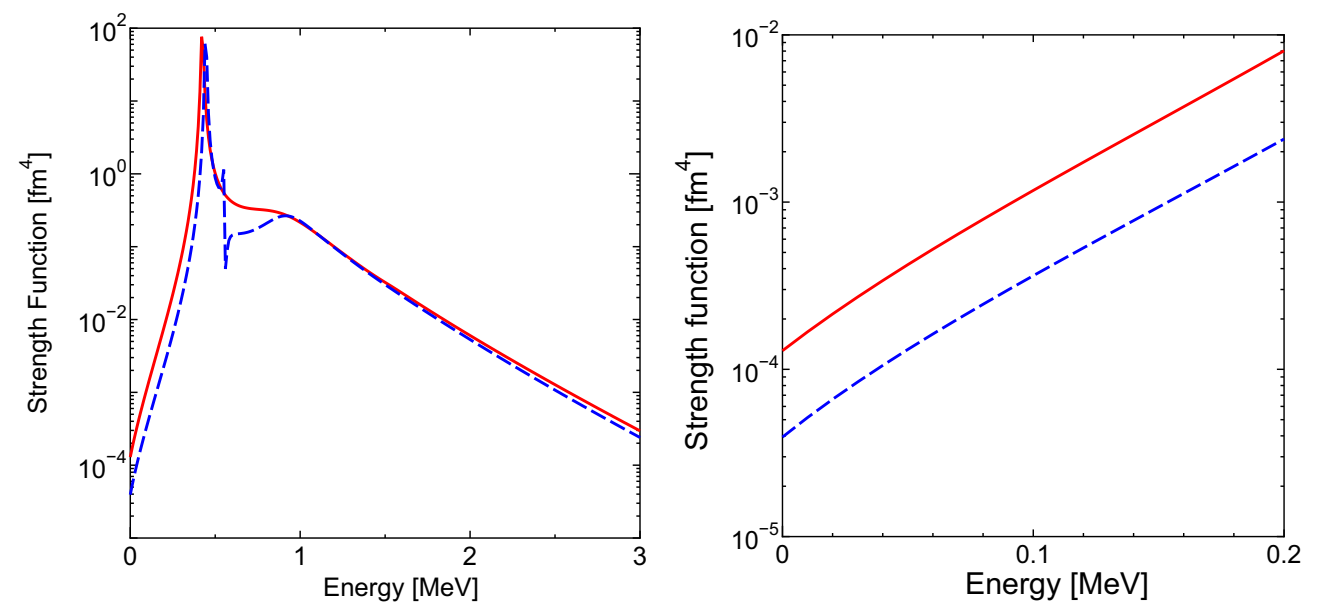

Figure 3. Left panel: Monopole strength functions calculated with and without the boson symmetry. These strengths are calculated by CSM. The solid curve shows the result with boson symmetry, while the dashed curve represent the strength without the symmetry. Right panel: Enlargement of the left panel in the low energy region.

the yield at the low energy side is sensitive to the treatment of the boson symmetry. The low energy strength is enhanced in the calculation with the boson symmetry, as shown by the solid curve. The enlargement of the strength in the low energy region is shown in the right panel of Fig. 3. We can clearly confirm that the strength with the symmetry is enhanced by a factor of three in comparison to the strength without the symmetry.

In recent studies, the reaction rate of the triple $\alpha$ reaction has been investigated by the sophisticated reaction theory $[13,14]$. In these calculations, the boson symmetry is neglected, and the model dependence of the reaction rate, such as the number of the channels and the spatial range of the calculation, is discussed in the order of magnitude of about $10^{20}$. The effect of the boson symmetry seems to be minor in comparison to the model dependence discussed in Refs. [13, 14]. However, the consideration of the boson symmetry will be important in the precise evaluation of the triple $\alpha$ reaction rate if the order of the magnitude is fixed appropriately.

\section{Summary}

In sum, we have formulated the method of the absorbing boundary condition (ABC) in the three-body problem with the coupled-rearrangement-channels variational method (CRCVM). The CRCVM + $\mathrm{ABC}$ has been applied to the schematic three-boson system, in which the boson symmetry is exactly considered by performing the rearrangement channels. We have introduced the absorbing boundary in two ways: the absorbing potentials in the Jacobi coordinate and the absorber in the particle pair coordinate. The resonance parameters calculated by these two methods are compared, and we have confirmed that the resonance parameters are the same value in these two methods.

The ABC results are also compared with the complex scaling method (CSM), which has already been successful in the application to the CRCVM calculation. The resonance parameters of CSM are consistent to the result of the $\mathrm{ABC}$ method although the decay width of the broad resonance in $\mathrm{ABC}$ is considerably different from that in the CSM calculation. This deviation is considered to be the 
reflection effect from the absorber. In the ABC method, the effect of the reflection cannot completely be eliminated even if the strength is optimized to minimize the numerical error. However, the deviation of the decay width is just the order of $10^{-3} \mathrm{MeV}$, in which the ambiguity in the theoretical model itself will be essential.

The strength function of the three-body breakup is also calculated by the ABC method plus the extended completeness relation (ECR). The strength of the ABC method nicely reproduces the CSM result. The decomposition of the strength functions is also investigated according to ECR. The individual contributions to the total strength are consistent to the CSM strength.

From the present comparison of ABC with CSM, we can conclude that the CRCVM + ABC method, formulated in the present article, nicely works in the three-body problem. The different ways of the absorber, such as the absorbers in the Jacobi coordinate and those in the pair coordinate, give the equivalent results in both the resonance parameters and the strength function if the strength of the absorbers are appropriately fixed. The computational number of the matrix elements is six in the Jacobi absorber (= 2 per channel $\times 3$ channels), while the number is three in the pair absorber (= pair number of three particles). Thus, the number of the matrix elements is reduced in the pair-coordinate absorber, which leads to save the computational time.

In the future subject, we should apply the $\mathrm{ABC}$ method to the more realistic system, such as the microscopic three cluster system with the full anti-symmetrization. The calculations of the microscopic cluster model can be easily done in the framework of the generator coordinate method (GCM) [16], but the application of CSM to GCM is still difficult [9]. On the contrary, the application of ABC to GCM has been successful in the two-body system [9]. Therefore, the application of ABC + GCM should be extended to the three-body system, such as the ${ }^{12} \mathrm{C}=3 \alpha$ system [17]. The application of $\mathrm{ABC}$ to the three-body GCM is now under progress.

\section{References}

[1] I. Tanihata, H. Savajols, and R. Kanungo, Prog. Part. Nucl. Phys. 68, 215 (2013).

[2] A. Deltuva, A. C. Fonseca, and P. U. Sauer, Ann. Rev. Nucl. Part. Sci. 58, 27 (2008).

[3] S. Aoyama, T. Myo, K. Katõ, and K. Ikeda, Prog. Theor. Phys. 116, 1-35 (2006), and references therein.

[4] T. Myo, K. Katõ, S. Aoyama, and K. Ikeda, Phys. Rev. C 63, 054313 (2001).

[5] S. Ohtsubo, Y. Fukushima, M. Kamimura, E. Hiyama, Prog. Theor. Exp. Phys. 2013, 073D02 (2013).

[6] U. V. Riss and H-D. Meyer, Jour. Phys. B26,4503 (1993).

[7] H. Masui and Y. K. Ho, Phys. Rev. C 65, 054305 (2002).

[8] M. Ueda et al., Phys. Rev. C 67, 014606 (2003).

[9] M. Ito and K. Yabana, Prog. Theor. Phys. 113, 1047 (2005).

[10] Y. Takenaka, et al., Prog. Exp. Theor. Phys. 2014, 113D04 (2014), and references therein.

[11] M. Iwasaki, et al., Prog. Exp. Theor. Phys. 2015 023D01, (2015).

[12] E. Hiyama et al., Prog. Part. Nucl. Phys. 51, 223 (2003).

[13] K. Ogata, M. Kan, and M. Kamimura, Prog. Theor. Phys. Vol. 122, 1055 (2009).

[14] T. Akahori, Y. Funaki, and K. Yabana, Phys. Rev. C92, 022801(R) (2015).

[15] G. Jolicard, C. Leforestier and E. J. Austin, Jour. Chem. Phys. 88, 1026, (1988).

[16] H. Horiuchi, Suppl. Prog. Theor. Phys. 62, 90 (1977).

[17] E. Uegaki, S. Okabe, H. Tanaka, Y. Abe, Prog. Theor. Phys. 57, 1262 (1977). 